\title{
On the eigenfunction expansion connected with the exterior problem for the Schrödinger equation
}

\author{
Teruo IKEBE \\ (Received October 10, 1966)
}

\section{Introduction.}

In this paper we concern ourselves with the study of differential operators of the form $-\Delta+q(x)$ in an exterior domain in 3-space with the Dirichlet boundary condition.

First we construct the Green functions for the Dirichlet problem in the case when $q(x)$ vanishes identically, following a work of Werner [11] $(\S 2)$. Then these Green functions will be employed to obtain the eigenfunctions of the form of plane wave plus outgoing wave satisfying the radiation condition at infinity, in terms of which an arbitrary square integrable function over the exterior domain can be expanded in an integral similar to the ordinary Fourier integral in 3 -space ( $\$ \S 3$ and 4$)$. The eigenfunction expansion problem of this sort has been investigated by Lax and Phillips [7], [8] and Shenk [9] in connection with the scattering theory for the wave equation in an exterior domain, and also by Shizuta [10]. One of the main purposes of this paper is to develop in detail the ideas due to Shizuta not provided with full proofs, though he has not used Werner's approach to the exterior Dirichlet problem but Mizohata's [6].

The Green functions for the $q=0$ case are a basis of all discussions that follow. The case of not identically vanishing $q(x)$ can be treated without any important alteration by the method developed in [2] which has dealt with the same problem in the whole 3-space, and therefore, we only indicate the tools and methods very briefly ( $\S 5,6$ and 7 ). However, we should mention here that under our assumption on $q(x)$ stated in $\S 5$ we have not succeeded in obtaining a proof of the orthogonality of the eigenfunctions used in the expansion theorem without the aid of the timedependent scattering theory (cf. $\S \S 8,9,10$ and 11 of [2]), but a more direct proof is possible under a stronger assumption on the asymptotic behavior of $q(x)$.

\section{$\S 1$. The exterior Dirichlet problem for $-\Delta$.}

Let $\Omega$ be an open domain $\subset R^{3}$ exterior to a bounded closed surface $\partial \Omega$ of class $C^{2}$. We consider the exterior Dirichlet problem of the following form :

(EDP) Let $f(x)$ be locally Hölder continuous in $\bar{\Omega}=\Omega \cup \partial \Omega$ and integrable over $\Omega$, and let $g(x)$ be a continuous function of $x \in \partial \Omega$. Find a function $u(x)$ which satisfies the following conditions:

i) $u(x)$ is continuous in $\Omega$ and twice continuously differentiable in $\Omega$;

ii) $u(x)$ satisfies the equation

$$
\Delta u+\kappa^{2} u=f \text { in } \Omega,
$$

where $\kappa$ is a complex constant with $\operatorname{Im} \kappa \geq 0$;

iii) $u(x)$ satisfies the boundary condition $u \mid \partial \Omega=g$;

iv) $u(x)$ satisfies the radiation condition at infinity

$$
u(x)=O(1 / r), \partial u(x) / \partial r-i \kappa u(x)=o(1 / r) \quad(r=|x| \rightarrow \infty)
$$


uniformly with respect to every direction.

We shall briefly describe Werner's results [11] on this problem. First we note that EDP can be reduced to the same problem with $f \equiv 0$ by the use of a suitably chosen volume potential with density $f(x)$.

Assume that $u(x)$, a solution to EDP, can be expressed as

$$
u(x)=S_{k} \sigma(x)+V_{\kappa} \tau(x),
$$

where

$$
\left\{\begin{aligned}
S_{\kappa} \sigma(x) & \left.=2 \int_{\partial \Omega} \sigma(y) \frac{\partial}{\partial n_{y}} F(x, y, \kappa) d S_{y}{ }^{*}\right) \\
V_{\kappa} \tau(x) & =-\int_{\omega} \tau(y) F(x, y, \kappa) d y
\end{aligned}\right.
$$

with

$$
F(x, y, \kappa)=\frac{e^{i \kappa|x-y|}}{4 \pi|x-y|} .
$$

Here $\sigma(x)$ and $\tau(x)$ are assumed to be continuous on $\partial \Omega$ and $\bar{\omega}=\omega \bigcup \partial \Omega$ respectively, $\omega$ being the domain interior to $\partial \Omega$, and $n_{x}$ denotes the outer normal (unit vector) to $\partial \Omega$ at $x$. In a standard way we obtain from (1.3)

$$
\sigma(x)+S_{\kappa} \sigma(x)+V_{k} \tau(x)=g(x) \text { on } \partial \Omega
$$

using the boundary condition $u \mid \partial \Omega=g$.

Introduce a function $\varphi(x)$ defined on $\bar{\omega}$ by

$$
\varphi(x)= \begin{cases}\eta_{0}{ }^{2} & \text { if } \operatorname{dist}(x, \partial \Omega)>\eta_{0} \\ \operatorname{dist}(x, \partial \Omega)^{2} & \text { if } \operatorname{dist}(x, \partial \Omega) \leq \eta_{0},\end{cases}
$$

$\eta_{0}$ being a positive constant sufficiently small but fixed in the sequel. Assume that $u(x)$, defined by (1.3) also for $x \in \omega$, satisfies

$$
\Delta u+\kappa^{2} u+i \varepsilon_{\kappa} \varphi(x) u(x)=0 \text { in } \omega,
$$

where $\varepsilon_{\kappa}=1$ or $=-1$ according as $\operatorname{Re} \kappa \geq 0$ or $\leq 0$ (in the case $\operatorname{Re} \kappa=0$ one may use either of $\varepsilon_{k}= \pm 1$ ). Then (1.8) together with (1.3) gives us, under some additional regularity assumption on $\tau(x)$,

$$
\tau(x)+i \varepsilon_{\kappa} \varphi S_{\kappa} \sigma(x)+i \varepsilon_{\kappa} \varphi V_{\kappa} \tau(x)=0(x \in \bar{\omega}) .
$$

That (1.9) has a meaning even on $\partial \Omega$ can be seen from the fact that $\varphi$ vanishes on $\partial \Omega$.

We note that equations (1.6) and (1.9) can be put in the matrix form as follows:

$$
X-K(\kappa) X=\Gamma
$$

with

$$
X=(\sigma, \tau), \Gamma=(g, 0),{ }^{* *)} K(\kappa) X=\left(\begin{array}{ll}
K_{11}(\kappa) & K_{12}(\kappa) \\
K_{21}(\kappa) & K_{22}(\kappa)
\end{array}\right) X
$$

where $\mathbb{K}_{j k}(\kappa)$ are defined by

*) $\left(\partial / \partial n_{x}\right) f(x)=\lim _{\tilde{o} \rightarrow 0}\left(\partial / \partial n_{x}\right) f\left(x+\delta n_{x}\right)$.

**) $(*, *)$ here and in what follows is used to denote the usual column vector $(*)$. 


$$
\begin{cases}K_{11}(\kappa) \sigma(x)=-S_{\kappa} \sigma(x) & (x \in \partial \Omega), \\ K_{12}(\kappa) \tau(x)=-V_{\kappa} \tau(x) & (x \in \partial \Omega), \\ K_{21}(\kappa) \sigma(x)=-i \varepsilon_{\kappa} \varphi S_{\kappa} \sigma(x) & (x \in \bar{\omega}), \\ K_{22}(\kappa) \tau(x)=-i \varepsilon_{\kappa} \varphi V_{\kappa} \tau(x) & (x \in \bar{\omega}) .\end{cases}
$$

Now let $B_{1}$ be the Banach space of all continuous functions $\sigma(x)$ on $2 \Omega$ with the norm $\|\sigma\|_{B_{1}}=\sup _{x \in \partial \Omega}|\sigma(x)|$ and $B_{2}$ the Banach space of all continuous functions $\tau(x)$ on $\bar{\omega}$ with the norm $\|\tau\|_{B_{2}}=\sup _{x \in \omega}|\tau(x)|$. Let $B$ be the Banach space of all pairs $X=(\sigma, \tau), \sigma \in B_{1}, \tau \in B_{2}$, normed by $\|X\|_{B}=\|\sigma\|_{B_{1}}+\|\tau\|_{B_{2}}$.

Then the operator $K_{j k}(\kappa)$ becomes a compact linear operator on $B_{k}$ to $B_{j}$, and thus $K(\kappa)$ is compact on $B$. One can now apply the Riesz-Schauder theory of compact operators in a Banach space to equation (1.10), and construct a solution of our EDP via (1.3). The uniqueness of an EDP solution can also be proved by a different method, and therefore we have

THEOREM 1.1. (Werner [11]). EDP has a unique solution of the form (1.3).

The above-mentioned results of Werner will be applied to construct and investigate properties of the Green functions for EDP. First we seek for the resolvent kernels for (1.10).

Define the operators $K_{j k}^{(n)}(\kappa)$ and $K^{(n)}(k)$ as follows:

$$
\left\{\begin{aligned}
K_{j k}^{(1)}(\kappa) & =K_{j k}(\kappa), K_{j k}{ }^{(n)}(\kappa)=\sum_{m}^{2}=1 K_{j m}(\kappa) K_{m k}{ }^{(n-1)}(\kappa), \\
K^{(n)}(\kappa) & =\left(\begin{array}{ll}
K_{11}{ }^{(n)}(\kappa) & K_{12}{ }^{(n)}(\kappa) \\
K_{21}{ }^{(n)}(\kappa) & K_{22}{ }^{(n)}(\kappa)
\end{array}\right) .
\end{aligned}\right.
$$

Obviously we have $K^{(n)}(\kappa)=[K(\kappa)]^{n}$ and each $K_{j k}^{(n)}(\kappa)$ is a compact integral operator from $B_{k}$ to $B_{j}$, whose kernel will be denoted by $K_{j k}^{(n)}(x, y, \kappa)$.

Lemma 1.1. $K_{j k}^{(1)}(x, y, \kappa)$ and $K_{j k}^{(2)}(x, y, \kappa)$ are continuous in $x$ and $y$ uniformily with respect to $\kappa$ in any compact domain $K$ of $\bar{C}^{+*}$ ) unless $x=y$, i.e. for any $\varepsilon>0$ and $\eta>0$ there exists a $\delta=\delta(\varepsilon, \eta)$ such that $\left|x_{1}-x_{2}\right| \leq \delta,\left|y_{1}-y_{2}\right| \leq \delta$ and $\left|x_{s}-y_{i}\right| \geq \eta$ $(s, t=1,2)$ imply $\left|K_{j k}{ }^{(n)}\left(x_{1}, y_{1}, \kappa\right)-K_{j k}{ }^{(n)}\left(x_{2}, y_{2}, \kappa\right)\right| \leq \varepsilon$, where $\delta$ is independent of $\kappa \in K$.

$K_{j k}^{(3)}(x, y, \kappa)$ is continuous in $x$ and $y$ uniformly with respect to $k$ in any compact domain $K$ of $\bar{C}^{+}$.

Moreover, we have the following estimates for $K_{j k}^{(n)}(x, y, k)$ :

$$
\begin{cases}\left|K_{1 k}^{(1)}(x, y, \kappa)\right| \leq C_{1} /|x-y| & (k=1,2), \\ \left|K_{1 k}^{(2)}(x, y, \kappa)\right| \leq C_{2}(1+|\log | x-y||) & (k=1,2), \\ \left|K_{2 k}^{(n)}(x, y, \kappa)\right| \leq C_{3} & (n=1,2 ; k=1,2) \\ \left|K_{j k}^{(3)}(x, y, \kappa)\right| \leq C_{4} & (j, k=1,2),{ }^{* *)}\end{cases}
$$

where the C's are constants independent of $\kappa$ in a compact domain of $\bar{C}^{+}$.

Proof. The assertions of the lemma for $K_{11}^{(1)}(x, y, n)$ are well-known in classical potential theory***) and those for the remaining $K_{j k}^{(1)}(x, y, \kappa)$ rather obvious.

First let us show the assertions for $K_{11}^{(2)}(x, y, k)$, the other $K_{j k}^{(2)}(x, y, k)$ being able to be handled similarly and more easily.

*) $C^{ \pm}=\{\kappa \mid \operatorname{Im} \kappa \gtrless 0\}, C_{ \pm}=\{\kappa \mid \operatorname{Re} \kappa \gtrless 0\}$ and - denotes closure.

**) $C_{j}(j=1,2, \cdots)$ here and in what follows will denote any constant whatsoever, absolute or not.

***) Cf. e.g. Kellogg [5]., Chap. VI. 
We have, using the estimates already established for $K_{j k}^{(1)}(x, y, \kappa)$,

$$
\begin{aligned}
\left|K_{11}{ }^{(2)}(x, y, \kappa)\right| \leq & \int_{\partial \Omega}\left|K_{11}(x, z, \kappa) K_{11}(z, y, \kappa)\right| d S_{z}+\int_{\omega}\left|K_{12}(x, z, \kappa) K_{21}(z, y, \kappa)\right| d z \\
\leq & {\left[\int_{\partial \Omega \cap B(y, a)}\left|K_{11}(x, z, \kappa) K_{11}(z, y, \kappa)\right| d S_{z}+\int_{\partial \Omega \cap B(y, a)^{c}}\right] } \\
& +C_{3} \int_{\omega}\left|K_{12}(x, z, \kappa)\right| d z \\
\leq & C_{1}{ }^{2} \int_{\partial \Omega \cap B(y, a)} \frac{d S_{z}}{|x-z||z-y|}+C_{5},
\end{aligned}
$$

where $B(y, a)$ is the ball of radius $a$ centered at $y$ and $B(y, a)^{c}$ its complement. Now $a$ can be chosen in the following fashion:*) For any $x_{0} \in \partial \Omega$ one can introduce a local rectangular coordinate system $\left(x_{1}, x_{2}, x_{3}\right)$ in a vicinity of $x_{0}$ with its origin $(0,0,0)$ at $x_{0}$ such that $\partial \Omega \cap B\left(x_{0}, a\right)$ can be represented as $x_{3}=f\left(x_{1}, x_{2}\right) \in C^{2}$, where the function $f$ satisfies

$$
\left\{\begin{array}{l}
f(0,0)=f_{x_{1}}(0,0)=f_{x_{2}}(0,0)=f_{x_{1} x_{2}}(0,0)=0 \\
\left|f\left(x_{1}, x_{2}\right)\right| \leq C_{6}\left(x_{1}{ }^{2}+x_{2}{ }^{2}\right) \\
f_{x_{1}}\left(x_{1}, x_{2}\right)^{2}+f_{x_{2}}\left(x_{1}, x_{2}\right)^{2} \leq C_{7}\left(x_{1}{ }^{2}+x_{2}{ }^{2}\right) \\
\left|n_{x}-n_{x_{0}}\right| \leq C_{8}\left|x-x_{0}\right|\left(x=\left(x_{1}, x_{2}, x_{3}\right)\right)
\end{array}\right.
$$

Here the choice of $a$ depends only on the geometry of $\partial \Omega$. If $|x-y|=d \leq a / 2$, then we have

$$
\begin{aligned}
\int_{\partial \Omega \cap B(y, a)} & \frac{d S_{z}}{|x-z||z-y|}=\int_{\partial \Omega \cap B(y, d / 2)}+\int_{\partial \Omega \cap B(x, d / 2)}+\int_{\partial \Omega \cap[B(y, a)-B(y, 3 d / 2)]}
\end{aligned}
$$

and under the integral sign, for instance, of the first integral on the right-hand side of (1.17), using (1.16),

$$
\begin{aligned}
& \frac{d S_{z}}{|x-z||z-y|} \leq \frac{\left[1+f_{z_{1}}\left(z_{1}, z_{2}\right)^{2}+f_{z_{2}}\left(z_{1}, z_{2}\right)^{2}\right]^{1 / 2}}{\left[\left(x_{1}-z_{1}\right)^{2}+\left(x_{2}-z_{2}\right)^{2}+\left(x_{3}-z_{3}\right)^{2}\right]^{1 / 2}} \cdot \frac{d z_{1} d z_{2}}{\left(z_{1}{ }^{2}+z_{2}{ }^{2}+z_{3}{ }^{2}\right)^{1 / 2}} \text { **) } \\
& \leq\left(1+C_{7} a^{2}\right)^{1 / 2} \frac{d z_{1} d z_{2}}{\left[\left(x_{1}-z_{1}\right)^{2}+\left(x_{2}-z_{2}\right)^{2}\right]^{1 / 2}\left[z_{1}^{2}+z_{2}^{2}\right]^{1 / 2}}
\end{aligned}
$$

with $x=\left(x_{1}, x_{2}, x_{3}\right), y=(0,0,0), z=\left(z_{1}, z_{2}, z_{3}\right)$. Now we can estimate each term of (1.17) easily and see that every term is bounded by a constant independent of $d$ except for the last, for which we have

$$
\int_{\partial \Omega \cap[B(y, a)-B(y, 3 d / 2)]} \leq C_{9}\left|\log \left(x_{1}^{2}+x_{2}^{2}\right)^{1 / 2}\right| .
$$

Since it can be seen in virtue of (1.16) that $d=\left(x_{1}{ }^{2}+x_{2}{ }^{2}+x_{3}{ }^{2}\right)^{1 / 2}$ and $\left(x_{1}{ }^{2}+x_{2}{ }^{2}\right)^{1 / 2}$ are mutually comparable, we can replace $\left(x_{1}^{2}+x_{2}^{2}\right)^{1 / 2}$ by $d$ in (1.19).

On the other hand, if $d>a / 2$, it is not hard to see that the left member of (1.17) is bounded by a constant independent of $x$ and $y$. Thus we have proved the second inequality of (1.14)

*) See e.g. Werner [11], p. 34 .

**) This inequality has only a symbolical meaning. 
Next let us prove the continuity of $K_{11}^{(2)}(x, y, \kappa)$ : we are to estimate

$$
\begin{aligned}
& \int_{\partial \Omega}\left[K_{11}\left(x_{1}, z, \kappa\right)-K_{11}\left(x_{2}, z, \kappa\right)\right] K_{11}(z, y, \kappa) d S_{z}, \\
& \int_{\omega}\left[K_{12}\left(x_{1}, z, \kappa\right)-K_{12}\left(x_{2}, z, \kappa\right)\right] K_{21}(z, y, \kappa) d z, \\
& \int_{\partial \Omega} K_{11}(x, z, \kappa)\left[K_{11}\left(z, y_{1}, \kappa\right)-K_{11}\left(z, y_{2}, \kappa\right)\right] d S_{z}, \\
& \int_{\omega} K_{12}(x, z, \kappa)\left[K_{21}\left(z, y_{1}, \kappa\right)-K_{21}\left(z, y_{2}, \kappa\right)\right] d z,
\end{aligned}
$$

where any $x$ is assumed to be distant from any $y$ by an amount not less than $\eta$.

For (1.20) we split the integral in three parts:

$$
(1.20)=\int_{\partial \Omega \cap B\left(x_{1}, r\right)}+\int_{\partial \Omega \cap B\left(x_{2}, r\right) \cap B\left(x_{1}, r\right)^{e}}+\int_{\text {rest }}=I_{1}+I_{2}+I_{3} .
$$

Then $I_{1}$ and $I_{2}$ can be made as small as we wish by choosing $r$ small enough in virtue of the first inequality of (1.14) and of the fact that $|z-y| \geq \eta-r$.

For the integrand of $I_{3}$, noting that $\left|x_{j}-z\right| \geq r(j=1,2)$, we have by a straightforward computation using the first inequality of (1.14)

$$
\left|\left[K_{11}\left(x_{1}, z, \kappa\right)-K_{11}\left(x_{2}, z, \kappa\right)\right] K_{11}(z, y, \kappa)\right| \leq C_{10} \frac{\left|x_{1}-x_{2}\right|}{r^{3}} \cdot \frac{1}{|z-y|},
$$

where $C_{10}$ depends on $\kappa$. Consequently $\left|I_{3}\right|$ is bounded by a constant multiple of $\left|x_{1}-x_{2}\right| / r^{3}$, which can be made arbitrarily small if $\left|x_{1}-x_{2}\right|$ is taken sufficiently small. Thus we see that for any $\varepsilon>0$ and $\eta>0$ there is a $\delta=\delta(\varepsilon, \eta)$ such that $\left|x_{1}-x_{2}\right| \leq \delta$ and $\left|x_{j}-y\right| \geq \eta$ imply that the absolute value of (1.20) is less than $\varepsilon / 4$, this estimate being uniform in $\kappa \in K$, a compact domain of $\bar{C}^{+}$.

For (1.22) it would be sufficient to note that for $\left|y_{1}-y_{2}\right|$ small enough and $\left|y_{j}-z\right| \geq r>0$ we can get by means of the last inequality of (1.16)

$$
\left|K_{11}\left(z, y_{1}, \kappa\right)-K_{11}\left(z, y_{2}, \kappa\right)\right| \leq C_{11}\left|y_{1}-y_{2}\right| / r^{3} .
$$

Then a similar estimate is possible for (1.22).

We can obtain similar results also for (1.21) and (1.23) by a simpler method and thus arrive at the required continuity of $K_{11}{ }^{(2)}(x, y, \kappa)$.

For the other $K_{j k}{ }^{(2)}(x, y, \kappa)$ the continuity can be proved more easily. Also we may remark that the condition $\left|x_{s}-y_{t}\right| \geq \eta$ is actually not involved in the continuity of $K_{2 k^{(2)}}(x, y, \kappa)(k=1,2)$.

$K_{j k}^{(3)}(x, y, \kappa)$ can be treated in much the same way, where one has of course to use the already established results about $K_{j k}^{(2)}(x, y, k)$. This concludes the proof of our lemma.

The following two lemmas concerning the regularity of various quantities related with $K(\kappa)$ are essentially due to Werner [11].

Lemma 1.2. $K_{j k}^{(n)}(x, y, \kappa)$ and $K^{(n)}(\kappa)$ are analytic in $\kappa$ in the whole complex plane $C$.

Lemma 1.3. $(I-K(\kappa))^{-1}$ is continuous in $K \in \bar{C}^{+} \cap \bar{C}_{+}\left[\bar{C}^{+} \cap \bar{C}_{-}\right]$and analytic in $\kappa \in \bar{C}^{+} \cap \bar{C}_{+}\left[\bar{C}^{+} \cap \bar{C}_{-}\right]$(in the uniform topology). 
Here we remark only that the proof of the analyticity asserted in the above lemmas can be carried through by means of power series expansion, while in order to show the continuity stated in Lemma 1.3 we need the fact that $(I-K(\kappa))^{-1}$ exists on the boundary (i.e. on the real and imaginary axes) of the domain considered.

Now let us define the function $R_{j k}(x, y, \kappa)$ by

$$
\begin{gathered}
\left(R_{11}(x, y, \kappa), R_{21}(z, y, \kappa)\right)=(I-K(\kappa))^{-1}\left(K_{11}{ }^{(3)}(x, y, \kappa), K_{21}{ }^{(3)}(z, y, \kappa)\right) \\
(x, y \in \partial \Omega, z \in \bar{\omega}), \\
\left(R_{12}(x, y, \kappa), R_{22}(z, y, \kappa)\right)=(I-K(\kappa))^{-1}\left(K_{12}{ }^{(3)}(x, y, \kappa), K_{22}{ }^{(3)}(z, y, \kappa)\right) \\
(x \in \partial \Omega, y, z \in \bar{\omega}),
\end{gathered}
$$

where the action of $(I-K(\kappa))^{-1}$ is on the variables $x$ and $z$, while the variable $y$ is kept fixed. $R_{j k}(x, y, \kappa)$ is well-defined, for $K_{j k}{ }^{(3)}(x, y, \kappa), y$ fixed, is a continuous function of $x$ belonging to $B_{1}$ or $B_{2}$.

Theorem 1.2. Let $\kappa \in \bar{C}^{+} \cap \bar{C}_{ \pm}$. Then

$$
(I-K(\kappa))^{-1}=I+K^{(1)}(\kappa)+K^{(2)}(\kappa)+R(\kappa),
$$

where $R(\kappa)$ is a compact integral operator on $B$ generated by $R_{j k}(x, y, \kappa)$.

$R_{j k}(x, y, \kappa)$ is continuous in $x$ and $y$ in their respective domains uniformly for $\kappa$ restricted to a compact domain $K \subset \bar{C}^{+} \cap \bar{C}_{ \pm} . R_{j k}(x, y, \kappa)$ and $R(\kappa)$ are analytic in $\kappa \in C^{+} \cap C_{ \pm}$and continuous in $\kappa \in \bar{C}^{+} \cap \bar{C}_{ \pm}$.

Proof. We shall first show the properties stated of $R_{j k}(x, y, \kappa)$ to hold. By Lemma 1.1 we have $\left(K_{1 k}{ }^{(3)}(x, y, \kappa), K_{2 k}^{(3)}(z, y, \kappa)\right) \in B$ strongly continuous in $y$ uniformly for $\kappa \in K$. Since $(I-K(\kappa))^{-1}$ is bounded, we see that $R_{j k}(x, y, \kappa)$ is continuous in $x$ and $y$ as asserted.

The continuity and analyticity in $\kappa$ of $R_{j k}(x, y, \kappa)$ follows from that of (I$K(x))^{-1}$ (Lemma 1.3) and of $K_{j k}^{(3)}(x, y, \kappa)$ (Lemma 1.2), which reasoning also applies to the corresponding statement for $R(\kappa)$.

It remains to prove (1.29). For this purpose it suffices to note that $R(\kappa)$ as defined by (1.29) is represented as

$$
R(\kappa)=(I-K(\kappa))^{-1} K^{(3)}(\kappa)
$$

and to show that this operator is generated by the kernel $R_{j k}(x, y, \kappa)$, whence follows the compactness of $R(\kappa)$. This, however, is an immediate consequence of the proposition that follows.

Proposition 1.1. Let $T$ be a bounded linear operator on $B$ and $K$ a linear integral operator on $B$ with a continuous kernel $K_{j k}(x, y)$. Then the product $T K$ is an integral (necessarily compact) operator with the kernel $H_{j k}(x, y)=T K_{j k}(x, y)$ with $T$ acting on the variable $x$.

Proof. We may assume without loss of generality that $K_{j k}(x, y)=0$ except when $j=k=1$. Let

$$
\left.\left(H_{11}(x, y), H_{21}(z, y)\right)=T\left(K_{11}(x, y), 0\right) \text { ( } y \text { fixed }\right) .
$$

Since $T$ is bounded and hence permutable with limiting operations, and since $K_{11}(x, y)$ can be approximated uniformly by a sequence of continuous functions of the form $\sum_{m=1}^{n} f_{m}(x) g_{m}(y)$, we have for any element $(\sigma, \tau)$ of $B$

$$
T K(\sigma, \tau)=T \lim _{n \rightarrow \infty} \sum_{m=1}^{n} \int_{\partial \Omega} g_{m}(y) \sigma(y) d S_{y}\left(f_{m}(x), 0\right)
$$




$$
=\lim _{n \rightarrow \infty} \sum_{m=1}^{n}\left(\int_{\partial \Omega} h_{m}{ }^{(1)}(x) g_{m}(y) \sigma(y) d S_{y}, \int_{\partial \Omega} h_{m}{ }^{(2)}(z) g_{m}(y) \sigma(y) d S_{y}\right),
$$

where we have put

$$
T\left(f_{m}, 0\right)=\left(h_{m}{ }^{(1)}(x), h_{m}{ }^{(2)}(z)\right)
$$

and we know that $\sum_{m=1}^{n} h_{m}^{(j)}(x) g_{m}(y)$ tends to $H_{j_{1}}(x, y)$ uniformly as $n \rightarrow \infty$. Therefore it follows that

$$
T K(\sigma, \tau)=\left(\int_{\partial \Omega} H_{11}(x, y) \sigma(y) d S_{y}, \int_{\partial \Omega} H_{21}(z, y) \sigma(y) d S_{y}\right),
$$

which was to be shown.

\section{$\S 2$. The Green function $G(x, y, n)$ for $-\Delta$.}

We are ready to define the Green function for the EDP explained in $\S 1$. Let $y$ be an arbitrary, but fixed for the moment, point in $\Omega=R^{3}-\bar{\omega}$. Let us put

$$
\Gamma=(-F(x, y, \kappa), 0)
$$

in (1.10). According to the preceding $\S$ there exists a unique solution of this equation (1.10), which we shall designate by

$$
X=(\sigma(x, y, \kappa), \tau(z, y, \kappa)) \quad(x \in \partial \Omega, z \in \bar{\omega}, y \in \Omega) .
$$

In view of Theorem 1.2 then we have

$$
\begin{gathered}
\sigma(x, y, \kappa)=-F(x, y, \kappa)-\int_{\partial \Omega}\left[\sum_{n=1}^{2} K_{11}^{(n)}(x, u, \kappa)+R_{11}(x, u, \kappa)\right] F(u, y, \kappa) d S_{u}, \\
\tau(z, y, \kappa)=-\int_{\partial \Omega}\left[\sum_{n=1}^{2} K_{21}^{(n)}(z, u, \kappa)+R_{21}(z, u, \kappa)\right] F(u, y, \kappa) d S_{u} .
\end{gathered}
$$

$$
\text { (2.3) and (2.4) in (1.3) we obtain }
$$

$$
H(x, y, \kappa)=S_{\kappa} \sigma(x, y, \kappa)+V_{\kappa} \tau(x, y, \kappa) \quad(x \in \Omega)
$$

and then put

$$
G(x, y, \kappa)=F(x, y, \kappa)+H(x, y, \kappa) .
$$

$G(x, y, \kappa)$ will be called the Green function for EDP in the sense that the function

$$
u(x)=\int_{\Omega} G(x, y, \kappa) f(y) d y
$$

furnishes us with the solution of EDP with the inhomogeneous term $f(x)$, a more precise account of which will be found below.

Theorem 2.1. i) $H(x, y, \kappa)$ can be extended continuously to be defined in $\bar{\Omega} \times \bar{\Omega} \times \bar{C}^{+}$unless $x=y$ on $\hat{o} \Omega$, whence follows the corresponding extension of $G(x, y, \kappa)$. These extensions will be denoted by $H(x, y, \kappa)$ and $G(x, y, \kappa)$ again.

ii) $H(x, y, \kappa)$ is continuous in $\bar{\Omega} \times \bar{\Omega} \times \bar{C}^{+}$unless $x=y \in \partial \Omega$. The same is true for $G(x, y, \kappa)$ if $x \neq y$.

iii) $H(x, y, \kappa)$ and $G(x, y, \kappa)$ are analytic functions of $\kappa \in C^{+}$.

iv) $\left(\partial / \partial n_{x}\right) H(x, y, \kappa)$ and $\left(\partial / \partial n_{x}\right) G(x, y, \kappa)$ exist and are continuous in $x \in \partial \Omega$ and in $y \in \Omega$.

v) $H(x, y, \kappa)$ and $G(x, y, \kappa)$ are symmetric in $x$ and $y$ whenever defined.

vi) For fixed $y \in \Omega H(x, y, \kappa)$ is a unique solution of the homogeneous EDP with 
the boundary condition $\left.H(x, y, \kappa)\right|_{x \in \partial \Omega}=-F(x, y, \kappa)$. Consequently $G(x, y, \kappa)$ satisfies the null boundary condition.

vii) Let $\kappa \in C^{+}$. Then

$$
\begin{aligned}
& \|H(\cdot, y, \kappa)\|_{L_{1}(\Omega)} \leq C_{12}, \quad\|G(\cdot, y, \kappa)\|_{L_{1}(\Omega)} \leq C_{13}, \\
& \|H(\cdot, y, \kappa)\|_{L_{2}(\Omega)} \leq C_{14},\|G(\cdot, y, \kappa)\|_{L_{2}(\Omega)} \leq C_{15},
\end{aligned}
$$

where the C's depend not on $y$ but only on $\kappa$.

viii) Let $\kappa \in C^{+}$. Let $f \in L_{2}(\Omega)$ and $u(x)$ be defined by(2.7). Then we have : a) $u(x)$ is bounded and continuous in $\Omega$ and $u \mid \partial \Omega=0$; b) $u \in H_{2}(\Omega)$, where $H_{2}(\Omega)$ is the set of all strongly twice differentiable functions $u(x)$ for which

$$
\|u\|^{2} H_{2}(\Omega)=\|u\|^{2} L_{2}(\Omega)+\sum^{3}{ }_{j=1}\left\|\frac{\partial u}{\partial x_{j}}\right\|_{L_{2}(\Omega)}^{2}+\sum_{j, k=1}^{3}\left\|\frac{\partial^{2} u}{\partial x_{j} \partial x_{k}}\right\|_{L_{2}(\Omega)}^{2}<\infty ;
$$

c) $u$ satisfies the equation

$$
-\Delta u-\kappa^{2} u=f,
$$

where $\Delta$ is taken in the sense of strong differentiation.

Proof of vi). Clear from the construction of $H(x, y, \kappa)$ and $G(x, y, \kappa)$.

Proof of iv). According to a result of Werner [11] (p. 47, (4.30)), $K_{j k}(\kappa)$ maps continuous functions into uniformly Hölder continuous functions. So, in view of (1.12), (1.6) and (1.9), $\sigma(x, y, \kappa)$ and $\tau(x, y, \kappa)$ are uniformly Hölder continuous, which implies in turn that $\sigma(x, y, \kappa)$ and $\tau(x, y, \kappa)$ are Hölder continuously differentiable, and iv) follows by virtue of (2.5) in a standard manner.*)

Proof of $\mathrm{v}$ ). If $x, y \in \Omega$, it is enough to consider the function

$$
G(z, y, \kappa) \Delta G(z, x, \kappa)-G(z, x, \kappa) \Delta G(z, y, \kappa)=0,
$$

to integrate it with respect to $z$ and to apply Green's formula. The domain of integration is at first a large ball intersecting $\Omega$ and excluding small balls about $x$ and $y$, and then a standard method may be followed. The treatment of the surface integrals can be carried through by use of the radiation condition on the one hand and of the already established results vi) and iv) on the other.

As will be shown later (the beginning part of the proof of i) and ii)), we can extend $H(x, y, \kappa)$ and $G(x, y, \kappa)$ without spoiling the symmetry.

Proof of vii). To start with we take up the $L_{1}$-estimate of $H(x, y, \kappa)$. Since we have by (2.5) and (1.4)

$$
\begin{aligned}
& \int_{\Omega}|H(x, y, \kappa)| d x \leq 2 \int_{\partial \Omega}|\sigma(z, y, \kappa)| d S_{z} \int_{\omega}\left|\frac{\partial}{\partial n_{z}} F(x, z, \kappa)\right| d x \\
& \quad+\int_{\omega}|\tau(z, y, \kappa)| d z \int_{\Omega}|F(x, z, \kappa)| d x
\end{aligned}
$$

and since the integrals $\int_{\Omega}\left|\left(\partial / \partial n_{z}\right) F(x, z, \kappa)\right| d x$ and $\int_{\Omega}|F(x, z, \kappa)| d x$ are bounded by a constant depending only on $\kappa$ but not on $z \in \partial \Omega$ and $\bar{\omega}$ respectively, we have only to verify that

*) These facts are more or less well-known in classical potential theory. For our purposes here see, e.g., [11], p. 36 . 


$$
\begin{aligned}
& \int_{\partial \Omega}|\sigma(z, y, \kappa)| d S_{z}, \\
& \int_{\omega}|\tau(z, y, \kappa)| d z
\end{aligned}
$$

have bounds dependent only on $\kappa$. In consideration of (2.3), (2.4) and (1.14) then it will turn out sufficient to show that

$$
\begin{gathered}
\int_{\partial \Omega}\left|R_{11}(z, u, \kappa)\right| d S_{z} \leq C_{16}, \\
\int_{(1)}\left|R_{21}(z, u, \kappa)\right| d z \leq C_{17},
\end{gathered}
$$

where $C_{16}, C_{17}$ are independent of $u$. But these are obvious from Theorem 1.2. Thus we have obtained (2.8).

For (2.9), using the Schwarz inequality, we have

$$
\begin{aligned}
\int_{\Omega}|H(x, y, \kappa)|^{2} d x \leq & C_{18} \int_{\Omega} d x \int_{\partial \Omega}|\sigma(z, y, \kappa)|^{2}\left|\frac{\partial}{\partial n_{z}} F(x, z, \kappa)\right| d S_{z} \int_{\partial \Omega} \frac{\partial}{\partial n_{z}} F(x, z, \kappa) \mid d S_{z} \\
& +C_{19} \int_{\partial \Omega} d x \int_{\omega} \mid \tau\left(z, y,\left.\kappa\right|^{2}|F(x, z, \kappa)| d z \int_{\omega}|F(x, z, \kappa)| d z\right. \\
\leq & C_{20}\left[\int_{\partial \Omega}|\sigma(z, y, \kappa)|^{2} d S+\int_{\omega}|\tau(z, y, \kappa)|^{2} d z\right] .
\end{aligned}
$$

With (2.3) and (2.4) in regard we can reduce the estimation of the last two integrals, by the same use of the Schwarz inequality as in (2.18), to that of

$$
\begin{aligned}
& \int_{\partial \Omega} d S_{z} \int_{\partial \Omega}|L(z, u, \kappa)| d S_{u} \int_{\partial \Omega}|L(z, u, \kappa)||F(u, y, \kappa)|^{2} d S_{u}, \\
& \int_{\omega} d z \int_{\partial \Omega}|M(z, u, \kappa)| d S_{u} \int_{\partial \Omega}|M(z, u, \kappa)||F(u, y, \kappa)|^{2} d S_{u}
\end{aligned}
$$

with $L=K_{11}{ }^{(1)}, K_{11}{ }^{(2)}, R_{11}$ and $M=K_{21}{ }^{(1)}, K_{21}{ }^{(2)}, R_{21}$. This may be easily done if we use Lemma 1.1 and Theorem 1.2 again, and then (2.9) follows.

The estimates for $G(x, y, \kappa)$ readily follow from those for $H(x, y, \kappa)$ and therefore we have accomplished the proof of vii).

Proof of i) and ii). If at least one of $x, y$ is on $\partial \Omega$ and if $x \neq y$, we define

$$
H(x, y, \kappa)=-F(x, y, \kappa) .
$$

This does not destroy the symmetry in $x$ and $y$, because the right side is symmetric. Moreover, we see that the continuity in $x$ [in $y]$ for fixed $y[x]$ of $H(x, y, \kappa)$ thus extended is preserved. So, in order to show that the asserted continuity is maintained, it is enough to verify the following: (1) $H(x, y, \kappa)$ is continuous in $x \in \bar{\Omega}$ uniformly with respect to $(y, \kappa)$ in a compact domain of $\Omega \times \bar{C}^{+} ;(2) H(x, y, \kappa)$ is continuous in $\kappa$ for fixed $x$ and $y$.

To prove (2) we consider for $x, y \in \Omega$ and for $\kappa, \kappa^{\prime} \in \overline{\boldsymbol{C}}^{+} \cap \overline{\boldsymbol{C}}_{ \pm}$, taking equation (2.5) into account, 


$$
\begin{aligned}
H(x, y, \kappa)-H\left(x, y, \kappa^{\prime}\right) & =\left(S_{\kappa}-S_{\kappa^{\prime}}\right) \sigma(x, y, \kappa)+S_{\kappa^{\prime}}\left[\sigma(x, y, \kappa)-\sigma\left(x, y, \kappa^{\prime}\right)\right] \\
& +\left(V_{\kappa}-V_{\kappa^{\prime}}\right) \tau(x, y, \kappa)+V_{\kappa^{\prime}}\left[\tau(x, y, \kappa)-\tau\left(x, y, \kappa^{\prime}\right)\right] .
\end{aligned}
$$

The first term on the right-hand side becomes

$$
2 \int_{\partial \Omega} \frac{\partial}{\partial n_{z}}\left[F(x, z, \kappa)-F\left(x, z, \kappa^{\prime}\right)\right] \sigma(z, y, \kappa) d S_{z} .
$$

Since $\sigma(z, y, \kappa)$ remains bounded for $z \in \partial \Omega$ when $\kappa$ varies over a compact set of $\bar{C}^{+} \cap \bar{C}_{ \pm}$as can be seen from (2.3) and Theorem 1.2, it follows at once that (2.23) can be made arbitrarily small by choosing $\kappa$ and $\kappa^{\prime}$ close enough to each other. The second term in full is

$$
2 \int_{\partial \Omega} \frac{\partial}{\partial n_{z}} F\left(x, z, \kappa^{\prime}\right)\left[\sigma(z, y, \kappa)-\sigma\left(z, y, \kappa^{\prime}\right)\right] d S_{z},
$$

which can also be made small by taking $\left|\kappa-\kappa^{\prime}\right|$ sufficiently small, similarly, by using Theorem 1.2. The third and fourth terms admit a similar handling.

If the compact set, in question, of $\bar{C}^{+}$intersects the imaginary axis, then we can argue as follows: The values of $H(x, y, \kappa), \operatorname{Re} \kappa=0$, viewed as the limits for $\operatorname{Re} \kappa \rightarrow 0$ \pm 0 , must coincide as a unique solution of one and the same EDP, and thus the continuity is guaranteed.

If $x$ or $y$ lies on $\partial \Omega$, then (2) is trivial.

Let us turn to (1). Consider

(2.25) $H(x, y, \kappa)-H\left(x^{\prime}, y, \kappa\right)=S_{\kappa}\left[\sigma(x, y, \kappa)-\sigma\left(x^{\prime}, y, \kappa\right)\right]+V_{\kappa}\left[\tau(x, y, \kappa)-\tau\left(x^{\prime}, y, \kappa\right)\right]$. Since the second term can be treated similarly to the first, we shall consider only the latter, which can be written as

$$
2 \int_{\partial \Omega} \frac{\partial}{\partial n_{z}}\left[F(x, z, \kappa)-F\left(x^{\prime}, z, \kappa\right)\right] \sigma(z, y, \kappa) d S_{z} .
$$

(We should remember here that we are dealing with the case when $x$ and $x^{\prime}$ are in $\Omega$, for otherwise (2.25) is not valid.)

Since $(y, \kappa)$ lies in a compact set of $\Omega \times \bar{C}^{+}, \sigma(z, y, \kappa)$ is bounded in $z \in \partial \Omega$ uniformly with resqect to $(y, \kappa)$, as we have already seen above. Thus we have

$$
|(2.26)| \leq \int_{\partial \Omega \cap B(x, r)}\left|\frac{\partial}{\partial n_{z}}\left[F(x, z, \kappa)-F\left(x^{\prime}, z, \kappa\right)\right]\right| d S_{z}+\int_{\partial \Omega B\left(x^{\prime}, r\right) \cap B(x, r)^{c}}+\int_{\text {rest }} .
$$

The first and second integrals can be made small by taking $r$ small enough, and the same can be done with the last if we note the inequality

$$
\left|\frac{\partial}{\partial n_{z}}\left[F(x, z, \kappa)-F\left(x^{\prime}, z, \kappa\right)\right]\right| \leq C_{21}\left|x-x^{\prime}\right| / r^{3} \quad\left(|x-z|,\left|x^{\prime}-z\right|>r\right)
$$

which can be obtained by an elementary computation (cf. (1.25)), where $C_{21}$ does not depend on how far $x$ and $x^{\prime}$ are away from $\partial \Omega$.

Thus $H(x, y, \kappa)$ is continuous in $x \in \Omega$ uniformly for $(y, \kappa)$ in a compact set of $\Omega \times \bar{C}^{+}$, and the convergence of $H\left(x^{\prime}, y, \kappa\right)$ to $-F(x, y, \kappa)$ for $x^{\prime} \rightarrow x \in \partial \Omega$ is uniform for $(y, \kappa)$ in the same compact set. This was to be shown.

Proof of iii). That $H(x, y, \kappa)$ is analytic in $C^{+} \cap C_{ \pm}$is immediate from Theorem 1.2, Lemma 1.2 and the definition of $H(x, y, \kappa)$. The analyticity in $C^{+}$is deduced from the continuity in $C^{+}$, which was established in the preceding proof. 
Proof of viii). $u(x),(2.7)$ for $f \in L_{2}(\Omega)$, is well-defined for every $x \in \Omega$ and bounded: $|u(x)| \leq\|G(x, \cdot, \kappa)\|_{L_{2}(\Omega)}\|f\|_{L_{2}(\Omega)}$.

For the proof of a) we consider

$$
\begin{aligned}
u\left(x_{1}\right)-u\left(x_{2}\right) & =\int_{\Omega \cap B\left(x_{1}, r\right)}\left[G\left(x_{1}, y, \kappa\right)-G\left(x_{2}, y, \kappa\right)\right] f(y) d y+\int_{\Omega \cap B\left(x_{2}, r\right) \cap B\left(x_{1}, r\right)^{\circ}}+\int_{\text {rest }} \\
& =J_{1}+J_{2}+J_{3} .
\end{aligned}
$$

For any $\varepsilon>0 r$ can be so chosen that $\left|J_{1}\right|<\varepsilon,\left|J_{2}\right|<\varepsilon$. It is not hard to show, by using the definition of $G(x, y, \tilde{x})$, that

$$
|G(x, y, \kappa)| \leq C_{22} \exp (-\operatorname{Im} \kappa|y|),
$$

if $x$ varies over a bounded domain and $|x-y|>r$. Therefore the application of the dominated convergence theorem enables us to get $\left|J_{3}\right|<\varepsilon$ if $\left|x_{1}-x_{2}\right|$ is sufficiently small. Thus we have proved the continuity.

$u \mid \partial \Omega=0$ being fulfilled is direct from (2.7) and vi).

The assertion b) is well-known, because the singularity involved in $G(x, y, \kappa)$ is nothing but that of the Newtonian potential. c) then follows immediately.

Now the proof of our theorem is complete.

\section{$\S 3$. The selfadjoint operator $H$ and the eigenfunctions $\varphi(x, k)$ for EDP.}

We shall define in this $\S$ a selfadjoint operator $H$ acting in the Hilbert space $L_{2}(\Omega)$ which is a restriction of $-\Delta$ in the broadest sense and which corresponds to the Dirichlet boundary condition on $\partial \Omega$, and introduce the generalized eigenfunctions associated with it.

Let $\kappa \in C^{+}$be purely imaginary so that $\kappa^{2}<0$. Define the operator

$$
G(\kappa) f(x)=\int_{\Omega} G(x, y, \kappa) f(y) d y .
$$

As is seen from Theorem 2.1 this operator is well-defined for $f \in L_{2}(\Omega)$. Moreover, the following assertion holds.

Lemma 3.1. Let $\kappa$ be as above. Then $G(\kappa)$ is a bounded selfadjoint operator on $L_{2}(\Omega)$.

Proof. That $G(\kappa)$ is bounded on $L_{2}(\Omega)$ can be seen directly as follows:

$$
\begin{array}{r}
\|G(\kappa) f\|^{2} \mathrm{~L}_{2}(\Omega) \leq \int_{\Omega} d x \int_{\Omega}|G(x, y, \kappa)| d y \int_{\Omega}|G(x, y, \kappa) \| f(y)|^{2} d y \\
\leq\left\|G\left(\cdot, y^{\prime}, \kappa\right)\right\| L_{L_{1}(\Omega)}\|G(x, \cdot, \kappa)\| L_{L_{1}(\Omega)}\|f\|^{2} L_{2}(\Omega) .
\end{array}
$$

Since we have seen that $G(\kappa)$ is bounded, we need only show that $G(\kappa)$ is symmetric for elements in a dense subset of $L_{2}(\Omega)$. Let, therefore, $f$ and $g$ be smooth $L_{2}(\Omega)$-functions so that $u=G(\kappa) f$ and $v=G(\kappa) g$ are smooth. Then $u$ and $v$ satisfy the equations (see Theorem 2.1, viii))

$$
\Delta u+\kappa^{2} u=-f, \Delta v+\kappa^{2} v=-g
$$

in the ordinary sense, so that we have

$$
(G(\kappa) f, g)_{L_{2}(\Omega)}=\left(u,\left[-\Delta-\kappa^{2}\right] v\right)_{L_{2}(\Omega)}=-(u, \Delta v)_{L_{2}(\Omega)}-\kappa^{2}(u, v)_{L_{2}(\Omega)} * *
$$

By means of integration by parts we can see that $(u, \Delta v)_{L_{2}(\Omega)}=(\Delta u, v)_{L_{2}(\Omega)}$ and hence

*) $(,)_{H}$ denotes the inner product in Hilbert space $H$. 


$$
(G(\kappa) f, g)_{L_{2}(\Omega)}=-(\Delta u, v)_{L_{2}(\Omega)}-\kappa^{2}(u, v)_{L_{2}(\Omega)}=(f, G(\kappa) g)_{L_{2}(\Omega)},
$$

which completes the proof.

Remark 3.1. As can be seen from the above proof $G(\kappa)$ for any $\kappa \in C^{+}$is a bounded linear operator on $L_{2}\left(S_{C}\right)$.

Lemma 3.2. $G(\kappa)^{-1}$ exists for any $\kappa \in C^{+}$and, in particular, is selfadjoint if $\kappa$ is purely imaginary. Furthermore, $D\left(G(\kappa)^{-1}\right)=R(G(\kappa))$ is independent of $\kappa \in C^{+}$.*)

PRoof. The first two assertions are obvious. To prove the last let

$$
\left.D=\left\{u: u \in H_{2}(\Omega)\right\}, u \text { is continuous in } \Omega, u \mid \partial \Omega=0\right\} .
$$

It is clear from Theorem 2.1, viii) that $R(G(\kappa)) \subset D$. For completing the proof, therefore, it is sufficient to verify the reverse inclusion, since $D$ is independent of $\kappa \in C^{+}$.

Let $u \in D$. If we put $f=-\Delta u-\kappa^{2} u$, then $f \in L_{2}(\kappa)$. Let us further put $v=u-G(\kappa) f$. Then $v$ satisfies

$$
\Delta v+\kappa^{2} v=0
$$

which implies that $v$ must be smooth. Since $v \in H_{2}(\Omega)$ and $v \mid \partial \Omega=0$, we have $0=\left(-\Delta v-\kappa^{2} v, v\right)_{L_{2}(\Omega)}=\|\operatorname{grad} v\|^{2} L_{2}(\Omega)-\kappa^{2}\|v\|^{2} L_{2}(\Omega)$, whence follows $v=0$ in view of the fact $\kappa \in C^{+}$. This shows $u=G(\kappa) f \in R(G(\kappa))$, which was to be proved.

THEOREM 3.1. There exists a unique nonnegative definite selfadjoint operator $H$ such that for every nonreal $\lambda$ the resolvent $R(\lambda ; H)=R(\lambda)=(H-\lambda I)^{-1}$ is equal to $G(\sqrt{\lambda})$, where by $\sqrt{\lambda}$ is meant the square root of $\lambda$ with nonnegative imaginary part, and hence $D(H)=D$ defined by (3.6).

Proof. The uniqueness is obvious. Now we need only define

$$
H=G(\kappa)^{-1}+\kappa^{2} I
$$

for some $\kappa \in C^{+}$. That $H$ is independent of $\kappa$ used in (3.8) can be seen by observing $D(H)=D$ and $H u=-\Delta u$ for $u \in D$. If $\kappa$ is purely imaginary, then we can see by Lemma 3.1 that $H$ is selfadjoint. Clearly $R\left(\kappa^{2}\right)=G(\kappa)$. Since every negative real lies in the resolvent set $\rho(H)$ of $H, H$ is necessarily nonnegative definite. Thus we have proved our theorem.

It is possible to verify that our $H$ coincides with the Friedrichs extension or the closure of the negative Laplacian defined for such $u$ that $u$ is an infinitely differentiable $L_{2}(\Omega)$-function vanishing on the boundary. We shall, however, never use this fact in the sequel.

Now let us proceed to the construction of the generalized eigenfunctions of $H$.

Let $k \in R^{3}$ and $\kappa \in C^{+}$. Consider the (conjugate) Fourier transform of the Green function $G(x, y, \kappa)$ :

$$
g(x, k, \kappa)=(2 \pi)^{-3 / 2} \int_{\Omega} G(x, y, \kappa) e^{i k \cdot y} d y,
$$

where $k \cdot y$ denotes the scalar product of $k$ and $y$. This definition makes sense, because $G(x, \cdot, \kappa) \in L_{1}(\Omega)$ (Theorem 2.1, vii)). Further we put

$$
\varphi(x, k, \kappa)=(2 \pi)^{3 / 2}\left(|k|^{2}-\kappa^{2}\right) g(x, k, \kappa) .
$$

Recalling that $F(x, y, \kappa)$ has the (conjugate) Fourier transform over $R^{3}$ equal to $(2 \pi)^{-3 / 2}\left(|k|^{2}-\kappa^{2}\right)^{-1} e^{i k \cdot x}$, we have

*) $D(A)$ and $R(A)$ denote the domain and range of operator $A$. 


$$
\begin{gathered}
\varphi(x, k, \kappa)=e^{i k \cdot x}+h(x, k, \kappa), \\
h(x, k, \kappa)=\left(|k|^{2}-\kappa^{2}\right)\left[\int_{\Omega} H(x, y, \kappa) e^{i k \cdot y} d y-\int_{\omega} G(x, y, \kappa) e^{i k \cdot y} d y\right] .
\end{gathered}
$$

A characterization of $h(x, k, k)$ is given by the following

Lemma 3.3. $h(x, k, k), k \in R^{3}, \kappa \in C^{+}$, is the unique EDP solution associated with the equation

$$
\left(\Delta+\kappa^{2}\right) h=0
$$

and the boundary condition

$$
h \mid \partial \Omega=-e^{i k \cdot x} .
$$

Moreover, $h(x, k, k)$ is continuous in $\bar{\Omega} \times R^{3} \times C^{+}$, and can be extended continuously to $\bar{\Omega} \times R^{3} \times \bar{C}^{+}$.

Proof. Let first $\kappa \in C^{+}$. That $h(x, k, \kappa)$ satisfies (3.13) and (3.14) and that $h(x, k, k)$ is twice continuously differentiable in $\Omega$ are a consequene of the definition (3.12) of $h(x, k, \kappa)$, Theorem 2.1 and the fact that $e^{i k \cdot x}$ is a nice function. One has, therefore, only to prove that it satisfies the radiation condition near infinity. In this connection we should note that the radiation condition cannot be satisfied by $g(x, k, \kappa)$.

The second integral of

$$
-\left(|k|^{2}-\kappa^{2}\right) \int_{\omega} G(x, y, \kappa) e^{i k \cdot y} d y
$$

is easily seen to satisfy the radiation condition, since $G(x, y, \kappa)$ does as a function of $x$ and since the integration is restricted to the bounded domain $\omega$.

Thus it remains to estimate

$$
\left(\frac{\partial}{\partial|x|}-i \kappa\right) \int_{\Omega} H(x, y, \kappa) e^{i k \cdot y} d y
$$

for sufficiently large $|x|$. In virtue of (2.5) and (1.4) we have

$$
\begin{aligned}
&\left|\left(\frac{\partial}{\partial|x|}-i \kappa\right) H(x, y, \kappa)\right| \leq 2 \max _{z \in \partial \Omega}\left|\left(\frac{\partial}{\partial|x|}-i \kappa\right) \frac{\partial}{\partial n_{z}} F(x, z, \kappa)\right| \int_{\partial \Omega}|\sigma(z, y, \kappa)| d S_{z} \\
&+\max _{z \in \omega}\left(\frac{\partial}{\partial|x|}-i \kappa\right) F(x, z, \kappa)\left|\int_{\omega}\right| \tau(z, y, \kappa) \mid d z \\
& \leq C_{23}|x|^{-2} e^{-\operatorname{Im} \kappa|x|} \int_{\partial \Omega}|\sigma(z, y, \kappa)| d S_{z}+C_{24}|x|^{-2} e^{-\operatorname{Im} \kappa|x|} \int_{\omega}|\tau(z, y, \kappa)| d z
\end{aligned}
$$

if $|x|$ is taken sufficiently large, where $C_{23}, C_{24}$ depend only on $\kappa$. Thus

$$
|(3.16)| \leq \frac{e^{-\operatorname{Im} \kappa|x|}}{|x|^{2}}\left[C_{23} \iint_{\partial \Omega}|\sigma(z, y, \kappa)| d S_{z} d y+C_{24} \iint_{\Omega}|\tau(z, y, \kappa)| d z d y\right]
$$

for $|x|$ large enough. Now by using (2.3) and (2.4) it is not hard to obtain the estimates

$$
\int_{\Omega}|\sigma(z, y, \kappa)| d y \leq C_{25}, \int_{\Omega}|\tau(z, y, \kappa)| d y \leq C_{26}
$$

uniform in $z \in \partial \Omega$ and $\in \omega$ respectively, where $C_{25}, C_{26}$ depend only on $\kappa$. (3.18) and (3.19) yield 


$$
|(3.16)| \leq C_{27} \frac{e^{-\operatorname{Im} \kappa|x|}}{|x|^{2}},
$$

whence the radiation condition being satisfied by $h(x, k, \kappa)$ is clear.

Now that we know the radiation condition being fulfilled, it is evident that $h(x, k, \kappa)$ solves uniquely the EDP with boundary data $-e^{i k \cdot x}, x \in \partial \Omega$. According to the argument in $\S 1$ which has led us to Theorem 1.1, if we put*)

$$
\begin{gathered}
\sigma(x, k, \kappa)=-e^{i k \cdot x}-\int_{\partial \Omega}\left[\sum^{2}{ }^{2}=1 K_{11}{ }^{(n)}(x, z, \kappa)+R_{11}(x, z, \kappa)\right] e^{i k \cdot z} d S_{z} \quad(x \in \partial \Omega), \\
\tau(x, k, \kappa)=-\int_{\partial \Omega}\left[\sum n^{2}=1 K_{21}{ }^{(n)}(x, z, \kappa)+R_{21}(x, z, \kappa)\right] e^{i k \cdot z} d S_{z} \quad(x \in \omega),
\end{gathered}
$$

$h(x, k, \kappa)$ can be represented as

$$
h(x, k, \kappa)=S_{\kappa} \sigma(x, k, \kappa)+V_{\kappa} \tau(x, k, \kappa) .
$$

From (3.21), (3.22) and the properties of $K_{j k}^{(n)}(x, y, \kappa)$ and $R_{j k}(x, y, \kappa)$ developed in $\$ 1$ (Lemmas $1.1,1.2,1.3$ and Theorem 1.2) it can be seen that the pair $(\sigma(\cdot, k, \kappa), \tau(\cdot, k, \kappa))$ is a $B$-valued continuous function of $k \in R^{3}$ and $\kappa \in \bar{C}^{+} \cap \bar{C}_{ \pm}$, since $\left(-e^{i k \cdot x}, 0\right)$ is so. Hence in view of $(3.23)$ the following two facts obtain : First, one can extend $h(x, k, \kappa)$, originally defined for $\kappa \in C^{+}$, to $\kappa \in \bar{C}^{+}$, and second, the function thus extended (which we shall denote by $h(x, k, \kappa)$ again) is continuous in $\bar{\Omega} \times R^{3} \times \bar{C}^{+}$. Here we have used the EDP uniqueness to continuate $h(x, k, \kappa)$ across $\operatorname{Re} \kappa=0$, as we have done in the proof of i) and ii) of Theorem 2.1. Now the proof of the lemma is complete.

Since $h(x, k, k)$ makes sense as seen above, we put

$$
\varphi(x, k)=\varphi(x, k,|k|)=e^{i k \cdot x}+h(x, k,|k|) .
$$

$\varphi(x, k)$ is uniquely determined by the requirement that the function $\varphi(x, k)-e^{i k \cdot x}$ be the unique solution of the homogeneous EDP for the equation

$$
\Delta h+|k|^{2} h=0 \text { in } \Omega
$$

and the boundary condition

$$
\left.h\right|_{\partial \Omega}=-e^{i k \cdot x},
$$

and the following theorem holds.

Theorem 3.2. There exists a family of generalized eigenfunctions $\varphi(x, k)$ $\left(\notin L_{2}(\Omega)\right)$ of $H$ satisfying the following conditions :

i) $\varphi(x, k)$ is continuous in $(x, k) \in \bar{z} \times R^{3}$.

ii) $\varphi(x, k), k \in R^{3}$ fixed, is twice continuously differentiable in $\Omega$, and satisfies the equation

$$
\Delta \varphi(x, k)+|k|^{2} \varphi(x, k)=0 \text { in } \Omega,
$$

the boundary condition

$$
\left.\varphi(x, k)\right|_{\partial \Omega}=0,
$$

and the function $h(x, k)=\varphi(x, k)-e^{i k \cdot x}$ satisfies the radiation condition

$$
h(x, k)=O\left(\frac{1}{|x|}\right), \quad\left(\frac{\partial}{\partial|x|}-i|k|\right) h(x, k)=o\left(\frac{1}{|x|}\right)(|x| \rightarrow \infty) .
$$

*) See also the beginning part of $\S 2$, where $H(x, y, \kappa)$ was constructed from (2.1). 
iii) $\varphi(x, k)$ is the boundary value at $\kappa=|k|$ of $\varphi(x, k, \kappa)=e^{i k \cdot x}+h(x, k, k)$, which is continuous in $\bar{a} \times R^{2} \times \bar{C}^{+}$and analytic in $\kappa \in C^{+}$and where $h(x, k, k)$ is the unique EDP solution for (3.13) and (3.14).

\section{$\S 4$. Eigenfunction expansions for $\#$.}

In this $\S$ we shall only outline how to get to the expansion of an arbitrary function $\in L_{2}(\Omega)$ in terms of the generalized eigenfunctions $\varphi(x, k)$ obtained in the preceding $\S$, because the method of proof employed here is essentially the same as that of [2].

Let $f \in C_{0}{ }^{\infty} C L_{2}(\Omega)$, and observe the well-known formula

$$
\begin{aligned}
\frac{1}{2}[(E(\beta) f & \left.+E(\beta-0) f, f)_{L_{2}(\Omega)}-(E(\alpha) f+E(\alpha-0) f, f)_{L_{2}(\Omega)}\right] \\
= & \frac{1}{2 \pi i} \lim \varepsilon \downarrow 0 \int_{\alpha}^{\beta}\left([R(\mu+i \varepsilon)-R(\mu-i \varepsilon] f, f) L_{2}(\Omega) d \mu,\right.
\end{aligned}
$$

where $E(\lambda)$ is the resolution of the identity, which is strongly continuous to the right, corresponding to the selfadjoint operator $H$.

Let us compute the integrand on the right-hand side of (4.1). Putting $\mu+i \varepsilon=$ $\kappa^{2}, \kappa \in C^{+}$, we have

$$
\begin{aligned}
\left(\left[R\left(\kappa^{2}\right)-R\left(\overline{\kappa^{2}}\right) f, f\right) L_{2}(\Omega)\right. & =\left(\kappa^{2}-\overline{\kappa^{2}}\right)\left(R\left(\kappa^{2}\right) f, R\left(\kappa^{2}\right) f\right) L_{2}(\Omega), \\
\left(R\left(\kappa^{2}\right) f, R\left(\kappa^{2}\right) f\right) L_{2}(\Omega) & =\int_{\Omega} d x \int_{\Omega} G(x, y, \kappa) f(y) d y \int_{\Omega} \overline{G(x, z, \kappa) f(z)} d z \\
& =\int_{\Omega} \int_{\Omega} f(y) \overline{f(z)} d y d z \int_{R^{3}} \overline{g(y, k, \kappa) g(z, k, \kappa)} d z,
\end{aligned}
$$

and finally, with (3.10) in regard,

$$
\begin{gathered}
(4.2)=\int_{R^{3}} \frac{2 i \varepsilon}{\left(|k|^{2}-\mu\right)^{2}+\varepsilon^{2}}|\Phi(k, \sqrt{\mu+i \varepsilon})|^{2} d k, \\
\Phi(k, \kappa)=(2 \pi)^{-3 / 2} \int_{0} \overline{\varphi(x, k, k) f(x) d x .}
\end{gathered}
$$

Here we have freely interchanged the integration order, which is guaranteed by THEOREM 2.1, vii).

Now we shall carry out the procedures indicated by formula (4.1). By interchanging $\lim$ and $\int$ on the right side of (4.1) and noting (4.4), we are led to the relation

$$
\begin{aligned}
\frac{1}{2}[(E(\beta) f & \left.+E(\beta-0) f, f) L_{2}(\Omega)-(E(\alpha) f+E(\alpha-0) f, f) L_{2}(\Omega)\right] \\
& = \begin{cases}\int_{\sqrt{\alpha} \leq|\kappa| \leq \sqrt{\beta}}|\hat{f}(k)|^{2} d k & (\alpha \geq 0) \\
0 & (\beta \leq 0),\end{cases}
\end{aligned}
$$

where we have put

$$
\hat{f}(k)=(2 \pi)^{-3 / 2} \int_{\dot{Q}} \varphi(x, k) f(x) d x=\lim _{\varepsilon \downarrow 0} \emptyset\left(k, \sqrt{|k|^{2}+i \varepsilon}\right),
$$


the existence of the limit in (4.7) following from Theorem 3.2. Here use has been made of the fact that

$$
\frac{1}{\pi} \frac{\varepsilon}{\left(|k|^{2}-\mu\right)^{2}+\varepsilon^{2}} \rightarrow \delta\left(|k|^{2}-\mu\right), \text { as } \varepsilon \rightarrow 0 .
$$

On the basis of (4.4) we can obtain

$$
([E(\beta)-E(\alpha)] f, g)_{L_{2}(\Omega)}=\int_{\sqrt{\alpha} \leq|k| \leq \sqrt{\beta}} \hat{f}(k) g(k) d k,
$$

where $0 \leq \alpha \leq \beta$. We can also see that $\hat{f} \in L_{2}\left(R^{3}\right)$ and $\|\hat{f}\|_{L_{2}\left(R^{3}\right)}=\|f\|_{L_{2}(\Omega)},(\hat{f}, g) L_{L_{2}\left(R^{3}\right)}$ $=(f, g)_{L_{2}(\Omega)}$. These results can be extended in an obvious way to the case $f, g \in L_{2}(\Omega)$. It is now easy to get a diagonal representation of $H$ :

$$
(H E(\lambda) f, g)_{L_{2}(\Omega)}=\int_{|k| \leq \sqrt{\lambda}}|k|^{2} \hat{f}(k) \overline{g(k)} d k \quad(\lambda \geq 0) .
$$

We already know that no spectrum is found on the negative line (Theorem 3.1). From (4.9) it is now clear that $(E(\lambda) f, f)$ is absolutely continuous with respect to the ordinary Lebesgue measure, which means that the absolutely continuous part of $H$ is $H$ itself. Moreover, since $E(0-0)=E(0)=0, H$ is strictly positive: $(H f, f) L_{2}(\Omega)$ $>0$ for $f \neq 0, \in D(H)$.

The inversion formula relative to (4.7) can also be easily deduced.

The mapping $Z$ that takes $f \in L_{2}(\Omega)$ into $\hat{f}(k) \in L_{2}\left(R^{3}\right)$ is isometric, but it is not obvious whether $Z$ maps onto $L_{2}\left(R^{3}\right)$. This, however, is the case, as we have shown in [3], and hence the inverse $Z^{-1}$ exists and is isometric, onto. From this it follows that a complete characterization of $D(H)$ is given by

$$
D(H)=\left\{f \in L_{2}(\Omega):|k|^{2} \hat{f}(k) \in L_{2}\left(R^{3}\right)\right\} .
$$

Now $H$ is clearly unitarily equivalent with $H^{0}$, the negative Laplacian in the whole $R^{3}$, whose precise definition may be given in terms of Fourier transforms as follows: (4.12) $D(H)=\left\{f \in L_{2}\left(R^{3}\right):|k|^{2} \hat{f}_{0}(k) \in L_{2}\left(R^{3}\right)\right\},\left(H^{0} f\right)_{0}(k)=|k|^{2} \hat{f}_{0}(k)$, where $\hat{f}_{0}(k)$ means the ordinary Fourier transform of $f \in L_{2}\left(R^{3}\right)$

$$
\hat{f}_{0}(k)=(2 \pi)^{-3 / 2} \text { 1.i.m. } \cdot_{R \rightarrow \infty} \int_{|x| \leq R} e^{-i k \cdot x} f(x) d x
$$

The results we have mentioned above are summarized in Theorems 7.1 and 7.2 , where we have only to put $q=0$, exclude any statement concerning the discrete eigenvalues, and note that $E_{0}(0)=E(0)=0$.

\section{§ 5. The Green functions and eigenfunctions for the exterior problem connected with $-\Delta+q(x)$.}

In this $\S$ we consider the differential operator $-\Delta+q(x)$ in $\Omega$ with null (Dirichlet) boundary condition on $\partial \Omega$ where $q(x)$ is the so-called potential function. Let us first state the assumption to be made on $q(x)$ :

(A) $q(x)$ is a realvalued function locally Hölder continuous in $\Omega$ except at a finite number of singularities, $\in L_{2}(\Omega)$ and satisfies

$$
|q(x)| \leq C|x|^{-2-h} \text { for }|x| \geq R_{0}
$$

where $C, h$ and $R_{0}$ are some positive constants. ${ }^{*}$

*) $\quad R_{0}$ is assumed to be so large that $\partial \Omega$ is contained in the interior of the ball $B\left(0, R_{0}\right)$. 
We shall construct a selfadjoint operator $H_{q}$ corresponding to $-\Delta+q(x)$ in $\Omega$ and the null boundary condition, which is connected in a natural way with $H$ defined in $\S 3$.

First let us show that under (A) we have

$$
\|q f\|_{L_{2}(\Omega)} \leq C_{28}\|H f\|_{L_{2}(\Omega)}+C_{29}\|f\|_{L_{2}(\Omega)} \text { for } f \in D=D(H),
$$

where $C_{28}, C_{29}$ are positive constants and in particular $C_{28}$ can be chosen arbitrarily small.

In fact we have for $f \in D$

$$
\begin{aligned}
\|q f\| L_{2}(\Omega) & =\int_{B(0, R) \cap \Omega}|q(x)|^{2}|f(x)|^{2} d x+\int_{B(0, R)^{c}} \\
& \leq \max |f(x)|^{2}\|q\| L_{L_{2}(\Omega)}+C_{30}\|f\|^{2} L_{2}(\Omega) \\
x \in B(0, R) \cap \Omega &
\end{aligned}
$$

with sufficiently large $R$. Since $f=G(\kappa)\left(H-\kappa^{2} I\right) f$ for $f \in D$, we can estimate $|f(x)|^{2}$ as follows:

$$
\begin{aligned}
|f(x)|^{2} & \leq \int_{\Omega}|G(x, y, \kappa)|^{2} d y \int_{\Omega}\left|\left(H-\kappa^{2} I\right) f(y)\right|^{2} d y \\
& \leq 2\left(\|H f\|^{2} L_{2}(\Omega)+|\kappa|^{4}|| f \|^{2} L_{2}(\Omega)\right)\|G(x, \cdot, \kappa)\|^{2} L_{2}(\Omega) .
\end{aligned}
$$

A re-examination of the estimates carried out for the proof of Theorem 2.1, vii) will give us the result that if $\kappa$ is, for example, purely imaginary and large enough, then $\|G(x, \cdot, \kappa)\|_{L_{2}(\Omega)}$ can be made as small as we wish independently of $x$. This together with (5.3) and (5.4) will lead us to (5.2) with $C_{28}$ arbitrarily small.

Having established this, we can prove the selfadjointness of the operator $-\Delta+q(x)$ with domain $D$ by showing that $(-\Delta+q(x) \pm i) f, f \in D$, form a dense set in $\left.L_{2}(\Omega) .{ }^{*}\right)$ Namely we have

Theorem 5.1. The operator $H_{q}$ defined by $D\left(H_{q}\right)=D(H)=D$ and $H_{q} f=H f+q f$, for $f \in D$, is selfadjoint and lower semibounded.

That $H_{q}$ is lower semibounded may be proved by the use of the inequality

$$
\left|(q f, f)_{L_{2}(\Omega)}\right| \leq C_{31}(H f, f) L_{L_{2}(\Omega)}+C_{32}(f, f) L_{L_{2}(\Omega)} \quad(f \in D)
$$

with an arbitrarily small constant $C_{31}$, which can be deduced from the integral representation

$$
f(x)=\int_{\Omega} \operatorname{grad}_{y} G(x, y, \kappa) \operatorname{grad} f(y) d y-\kappa^{2} \int_{\Omega} G(x, y, \kappa) f(y) d y
$$

of $f \in D$ by a method similar to that by which we obtained (5.3) and (5.4).**)

In the sequel we shall state most of the results without proof, because they and their proofs are analogous to those of [2] for the whole $R^{3}$ case. We consider $H_{q}$ as the result of applying the perturbing operator of multiplication by $q(x)$ to the unperturbed operator $H=H_{0}(q=0)$. Various quantities related with $q(x)$ or $H_{q}$ will be denoted by the corresponding quantities for the case $q=0$ with the subscript $q$.

Theorem 5.2.***) Let $\kappa \in C^{+}$and $\operatorname{Im} \kappa^{2} \neq 0$. Then $\kappa^{2} \in \rho_{q}=\rho\left(H_{q}\right)$, the resolvent set

*) Cf. Kato [4].

**) Cf. also Birman [1] and Kato [4].

***) Cf. [2], Theorems 1,2, Lemmas 2.1 and 9.1 . 
of $H_{q}$, and $R_{q}\left(\kappa^{2}\right)=\left(H_{q}-\kappa^{2} I\right)^{-1}$ is an integral operator of Carleman type with kernel $G_{q}(x, y, \kappa)$ satisfying the following conditions:

a) $G_{q}(x, y, \kappa)$ is a continuous function of $x \in \bar{\Omega}$ except at $x=y$ for each fixed $y \in \bar{\Omega}$;

b) $G_{q}(x, y, \kappa)=G_{q}(y, x, \kappa)(x \neq y)$;

c) $G_{q}(x, y, k)$ satisfies the integral equation

$$
G_{q}(x, y, \kappa)=G(x, y, \kappa)-\int_{\Omega} G(x, z, \kappa) q(z) G_{q}(z, y, \kappa) d z
$$

as a function of $x \in \bar{\Omega}(x \neq y)$ for fixed $y \in \bar{\Omega}$;

d) For fixed $\left.y \in \bar{\Omega} G_{q}(x, y, \kappa)\right|_{\partial \Omega}=0(x \neq y)$;

e) $\left\|G_{q}(\cdot, y, \kappa)\right\|_{L_{2}(\Omega)} \leq C_{33}$ and $\left\|G_{q}(\cdot, y, \kappa)\right\|_{L_{1}(\Omega)} \leq C_{34}$, where $C_{33}$ and $C_{34}$ are constants depending only $\kappa$;

f) $G(x, y, \kappa)$ is characterized as a unique solution of (5.7) belonging to $L_{2}(\Omega)$ for each fixed $y$;

g) $G(x, y, \kappa)$ is expressible as

$$
G(x, y, \kappa)=\sum_{j=0}^{3} A^{(j)}(x, y, \kappa)+G_{q}{ }^{\prime}(x, y, \kappa),
$$

where

$$
\begin{aligned}
& A^{(0)}(x, y, \kappa)=G(x, y, \kappa), \\
& A^{(j)}(x, y, \kappa)=-\int_{\Omega} G(x, y, \kappa) q(z) A^{(j-1)}(z, y, \kappa) d z \quad(j \geq 1),
\end{aligned}
$$

(which are defined also for any $\kappa \in \bar{C}^{+}$) and $G_{q}{ }^{\prime}(x, y, \kappa)$ is a unique solution in $E$ for fixed $y \in \Omega$ and for $\kappa \in C^{+}$) of

$$
G_{q}{ }^{\prime}(x, y, \kappa)=A^{(4)}(x, y, \kappa)-\int_{\Omega} G(x, z, \kappa) q(z) G_{q}{ }^{\prime}(z, y, \kappa) d z,
$$

$E$ being the Banach space of all continuous functions $u(x)$ tending to 0 uniformly as $|x|$ tends to infinity and satisfying $\left.u\right|_{\partial \Omega}=0$, normed by

$$
\|u\|_{E}=\max _{x \in \Omega}|u(x)|,
$$

and where $A^{(j)}(x, y, n)$ are continuous in $x \in \Omega$ for $x \neq y \in \Omega$, satisfying the boundary condition $\left.A^{(j)}(x, y, \kappa)\right|_{\partial \Omega}=0$ with $y \in \Omega$ fixed, and in particular $A^{(4)}(\cdot, y, \kappa) \in E$.

We can deduce (5.7) valid a.e. in $x$ for a.e. fixed $y \in \Omega$ from the second resolvent equation for $R(\lambda)$ and $R_{q}(\lambda)$ as we have done in [2], $\S 1$. This combined with a) gives c), while the proof of $b$ ) is exactly the same as in [2] if we assume a) to be true. d) is almost trivial. c) and f) can be proved as in [2] (see $\S \S 2,5$, Lemmas 2.3 and 9.1).

a) is a direct consequence of $\mathrm{g}$ ), and in order to prove $\mathrm{g}$ ) the following lemma will be useful.

Lemia 5.1. $H(x, y, \kappa)$ admits the estimate

$$
|H(x, y, \kappa)| \leq C_{35} /(1+|x-y|),
$$

where $C_{35}$ may depend on $\kappa \in \bar{C}^{+}$.

The proof of the above lemma is rather straightforward. It may be enough to note the definition of $H(x, y, x)$ in $\S 2$, Lemma 1.1 and Theorem 1.2.

It is now immediate to see that $A^{(0)}(x, y, \kappa)=G(x, y, \kappa)$ is continuous for $x \neq y$ 
and satisfies the boundary condition. Using Lemma 5.1 we can then estimate $A^{(1)}(x, y, \kappa)$ as

$$
\left|A^{(1)}(x, y, \kappa)\right| \leq C_{36}|x-y|^{-1 / 2}
$$

Similarly we may have

$$
\begin{aligned}
\left|A^{(1)}(x, y, \kappa)\right| & \leq C_{37} & & (|x-y| \geq 1 / 2) \\
& \leq C_{38} \log (1 /|x-y|) & & (|x-y|<1 / 2) \\
\left|A^{(j)}(x, y, \kappa)\right| & \leq C_{39}(j=3.4) . & &
\end{aligned}
$$

(For these estimates cf. [2], Lemma 2.2.). The continuity asserted in $g$ ) of $A^{(j)}(x, y, \kappa)$ can now be proved exactly as in $\S 5$ of [2]. The boundary condition to be satisfied by $A^{(j)}(x, y, \kappa)$ is obvious from construction.

That $A^{(4)}(\cdot, y, \kappa) \in E$ is a consequence of the following proposition whose proof is the same as for Lemma 4.1 of [2].

Proposition 5.1. Let

$$
T(\kappa) u(x)=-\int_{Q} G(x, y, \kappa) q(y) u(y) d y .
$$

Then for any bounded function $u(x)$ defined on $\Omega, T(\kappa) u(x)$ is an $E$-function Hölder continuous with exponent $1 / 2$ and satisfying the boundary condition $\left.T(\kappa) u(x)\right|_{\partial \Omega}=0$.

The only point in this proposition that differs from Lemma 4.1 and the remark to it of [2] is the boundary values on $\partial 2$, which, however, offers no difficulty. So, a similar proof is possible if we take account of Lemma 5.1 .

To complete the proof of $g$ ) of Theorem 5.2 we need another proposition concerning the existence and uniqueness of the solution to the equation

$$
u(x)=f(x)-\int_{\Omega} G(x, y, \kappa) q(y) u(y) d y \text { or } u=f+T(\kappa) u \text {. }
$$

The study of (5.17) for $f \in E$ can be reduced, in virtue of the following proposition which asserts the complete continuity of $T(\kappa)$, to that of the associated homogeneous equation

$$
u(x)=-\int_{\Omega} G(x, y, \kappa) q(y) d y \text { or } u=T(\kappa) u
$$

Proposition 5.2. $T(\kappa)$ is a compact (completely continuous) linear operator on $E$. The proof is similar to that of Lemma 4.2 of [2].

Proposition 5.3. Let $\kappa \in C^{+}$. Then (5.18) has nontrivial solutions in $E$ if and only if $\kappa^{2}$ is an eigenvalue of $H_{q}$. In particular, if $\operatorname{Im} \kappa^{2} \neq 0$ in addition, then (5.18) has only the trivial solution in $E$.

Proposition 5.4. Let $\kappa$ be real and $\kappa \neq 0$. Then (5.18) has no solution other than $u=0$.

The proof of the above two propositions can be given in a way similar to Lemmas 4.5 and 4.4 of [2], while we should note that the proof of Proposition 5.4 is based upon the investigation of the asymptotic behavior of the function of the form

$$
w(x)=-\int_{\Omega} G(x, y, \kappa) v(y) d y
$$

for which we have the following four propositions. 
Proposition 5.5. Let $\kappa \in \bar{C}^{+}, v(x)$ be locally integrable and $v(x)=0\left(|x|^{-2-\infty}\right)$ $(|x| \rightarrow \infty)$ with $\varepsilon>0$. Then as $|x| \rightarrow \infty$

$$
w(x)=0\left(|x|^{-1}\right)+0\left(|x|^{-\varepsilon}\right) .
$$

Proposition 5.6. Let $\kappa$ be real, $v \in L_{2}(\Omega)$ and $v(x)=0\left(|x|^{-3-\varepsilon}\right)(|x| \rightarrow \infty)$. Then as $|x| \rightarrow \infty$

$$
w(x)=\frac{e^{i x|x|}}{|x|} b\left(\frac{x}{|x|}\right)+0\left(|x|^{-1-\varepsilon / 2}\right)+0\left(|x|^{-2}\right),
$$

where $b$ is some continuous function on the unit sphere of $R^{3}$.

Proposition 5.7. Let $v(x)$ be as in Proposition 5.6 and let $\kappa \neq 0$ be in $\bar{C}^{+}$. Then $w(x)$ satisfies the radiation condition.

Proposition 5.8. Let $v \in E$ be a solution of (5.18), where $\kappa$ is real and nonzero. Then $b$ in Proposition 5.6 is identically equal to zero.

These four propositions are analogous to Lemmas 3.1 to 3.4 of [2] and may be proved similarly.

Now from Propositions 5.3 and 5.4 we obtain

Proposition 5.9. Assume that $\kappa \neq 0$ is real or that $\kappa \in \bar{C}^{+}$is such that $\kappa^{2}$ is no eigenvalue of $H_{q}$. Then (5.17) has a unique solution $u$ in $E$ for any $f \in E$.

The next proposition concerns the dependence on the parameter $\kappa$ of $T(\kappa)$ and is essentially not different from the contents of Lemmas 4.6 and 4.7 of [2].

Proposition 5.10. $T(\kappa)$ is a continuous function of $\kappa \in \bar{C}^{+}$in the uniform topology and $(I-T(\kappa))^{-1}$ is also cotinuous in $\kappa$ if $\kappa \neq 0$ and $\kappa^{2}$ is no negative eigenvalue of $H_{q}$.

From this follows

Proposition 5.11. Let $K \subset \bar{C}^{+}$be a compact domain not containing the origin and any point whose square is a negative eigenvalue of $H_{q}$. Let $u(\kappa)$ be the unique solution of

$$
u(\kappa)=f(\kappa)+T(\kappa) u(\kappa),
$$

where $f(\kappa) \in E$ is strongly continuous in $\kappa \in K$. Then $u(\kappa)$ is strongly continuous in $\kappa \in K$.

By means of Proposition 5.9 we can complete the proof of Theorem 5.2, g) as in $\S 5$ of [2].

Now let us turn to the construction of the generalized eigenfunctions of $H_{q}$. Consider the Lippmann-Schwinger equation

$$
\varphi_{q}(x, k)=\varphi(x, k)-\int_{\Omega} G(x, y,|k|) q(y) \varphi_{q}(y, k) d y .
$$

Since $\varphi(x, k)$ does not lie in $E$ (see Theorem 3.2), let us introduce

$$
p_{q}(x, k)=-\int_{a} G(x, y,|k|) q(y) \varphi(y, k) d y
$$

and make a substitution

$$
\varphi_{q}(x . k)=\varphi(x, k)+h_{q}(x, k)
$$

in (5.23), which gives us the equation

$$
h_{q}(x, k)=p_{q}(x, k)-\int_{\Omega} G(x, y,|k|) q(y) h_{q}(y, k) d y .
$$


Since it follows from Proposition 5.1 and the boundedness of $\varphi(x, k)$ (Theorem 3.2) that $p_{q}(x, k)$ belongs to $E$ for each $k \in R^{3}$, equation (5.26) has a unique solution $h_{q}(x, k) \in E$ according to Proposition 5.9.

This is also the case with the function $p_{q}(x, k, \kappa)$ defined by

$$
p_{q}(x, k, \kappa)=-\int_{\Omega} G(x, y, \kappa) q(y) \varphi(y, k) d y \quad\left(\kappa \in \bar{C}^{+}\right)
$$

and the corresponding solution $h_{q}(x, k, \kappa)$ of

$$
\left.\left.h_{q}(x, k, \kappa)=p_{q}(x, k, \kappa)-\int_{\Omega} G(x, y, \kappa) q\right) y\right) h_{q}(y, k, \kappa) d y \quad\left(\kappa \in \bar{C}^{+}\right) .
$$

Lemma 5.2. Let $K_{k}$ and $K_{k}$ be compact domains of $R^{3}$ and $\bar{C}^{+}$respectively. Then $p_{q}(x, k, \kappa)$ is uniformly continuous on $\bar{\Omega} \times K_{k} \times K_{k}$ and $p_{q}(x, k,|k|)=p_{q}(x, k)$.

This may be proved similarly to Theorem 3 and Lemma 9.2 of [2]. We need only take into account the estimate for $H(x, y, \kappa)$ stated in Lemma 5.1.

Lemma 5.2 together with the fact that $p_{q}(x, k, k) \in E$ for each fixed $k$ and $k$ implies that $p_{q}(\cdot, k, \kappa)$ is strongly continuous in $(k, \kappa) \in K_{k} \times K_{k}, K_{k}$ and $K_{k}$ being as in Lemma 5.2. Therefore, in view of Proposition 5.11, we have

Lemma 5.3. The unique solution $h_{q}(\cdot, k, \kappa) \in E$ of $(5.28)$ is a strongly continuous function of $(k, \kappa) \in K_{k} \times K_{k}$, with $K_{k}$ as in Lemma 5.2 and $K_{k}$ as $K$ in Proposition 5.11, and $h_{q}(x, k,|k|)=h_{q}(x, k)$.

Lemma 5.4. For $\kappa \in C^{+}, \operatorname{Im} \kappa^{2} \neq 0$, we have

$$
\varphi_{q}(x, k, \kappa)=\varphi(x, k)+h_{q}(x, k, \kappa)=(2 \pi)^{3 / 2}\left(|k|^{2}-\kappa^{2}\right) g_{q}(x, k, \kappa),
$$

where

$$
g_{q}(x, k, \kappa)=(2 \pi)^{-2 / 2} \int_{\Omega} G_{q}(x, y, \kappa) \varphi(y, k) d y \equiv Z G(x, \cdot, \kappa)(k)
$$

is a well-defined bounded function of $x$.

For this lemma the last assertion is immediate from Theorem 5.2, e) and Theorem 3.2. To prove (5.29) we have only to show that $g_{q}(x, k, \kappa)$ satisfies

$$
g_{q}(x, k, \kappa)=(2 \pi)^{-3 / 2} \frac{\varphi(x, k)}{|k|^{2}-\kappa^{2}}-\int_{\Omega} G(x, y, \kappa) q(y) g_{q}(y, k, k) d y,
$$

which can be done as in the proof. of Lemma 9.2 of [2], starting from the integral equation (5.7) satisfied by $G_{q}(x, y, \kappa)$ and using the fact that

$$
\int_{\Omega} G(x, y, \kappa) \varphi(y, k) d y=\frac{\varphi(x, k)}{|k|^{2}-\kappa^{2}},
$$

as can be verified by means of the eigenfunction expansion for $H$ discussed in $\S 4$.

Now in view of (5.23) and the Hölder continuity of $q(x)$ we can see that $\varphi_{q}(x, k)$ is continuous in $\bar{\Omega}$ and twice continuously differentiable in $\Omega$, except at the singularities of $q(x)$ satisfies

$$
-\Delta \varphi_{q}(x, k)+q(x) \varphi_{q}(x, k)=|k|^{2} \varphi_{q}(x, k)
$$

and fullfils the null boundary condition on $\partial \Omega$. Summarizing we have

THEorem 5.3. There exists a family of generalized eigenfunctions $\varphi_{q}(x, k)$ of $H_{q}$ having the following properties.

i) $\varphi_{q}(x, k)$ is continuous in $\bar{\Omega} \times\left(R^{3}-\{0\}\right)$ 
ii) $\varphi_{q}(x, k), k \in K^{3}-\{0\}$ fixed, is twice continuously differentiable in $\Omega$ except at the singularities of $q(x)$ and satisfies

(5.33) as well as the boundary condition $\left.\varphi_{q}(x, k)\right|_{\partial \Omega}=0$.

iii) $\varphi_{q}(x, k)$ is the boundary value at $\kappa=|k|$ cf $\varphi_{q}(x, k, \kappa)=\varphi(x, k)+h_{q}(x, k, \kappa)$, which is continuous in $\bar{\Omega} \times\left(R^{3}-\{0\}\right) \times \bar{C}^{+}$and where $h_{q}(x, k, \kappa)$ is the unique solution in $E$ of (5.28), and is characterized as a unique solution of (5.23) in the sense specified above.

\section{§6. The spectrum of $\boldsymbol{H}_{q}$.}

We have already seen that $H_{q}$ is lower semibounded, but we can say more concerning the spectrum of $H_{q}$. The following result is due to Birman [1] and we omit the proof thereof, only emphasizing that an essential role is played by the fact that $q(x)$ behaves like $0\left(|x|^{-2-h}\right)$ near infinity.

Theorem 6.1. The essential spectrum of $H_{q}$ is $[0 . \infty)$ and the spectrum on the negative reals consists of a finite number of eigenvalues of finite multiplicity; more precisely, $E_{q}(0-0) L_{2}(\Omega)$ is a finite-dimensional subspace, where $E_{q}(\lambda)$ denotes the right-continuous resolution of the identity associaited with $H_{q}$.

We can say even more about the essential spectrum of $H_{q}$ as a result of the eigenfunction expansion theorem to be discussed in the next $\S$. The point 0 , however, remains unclear in our arguments; it may or may not be an eigenvalue.

The discrete negative eigenvalues and 0 , if it is an eigenvalue, enumerated according to multiplicity will be denoted by $\mu_{q}^{(n)}, n=1.2, \cdots \cdots, N$ ( $N$ may be infinite only if 0 is an ligenvalue), and the associated eigenvectors $\in L_{2}(\Omega)$ by $\varphi_{q}^{(n)}(x)$.

\section{$\S 7$. Eigenfunction expansion for $H_{q}$.}

Let $f \in L_{2}(\Omega)$. Then the functions

$$
\begin{array}{r}
\hat{f}_{q, R}(k)=(2 \pi)^{-3 / 2} \int_{\Omega \cap B(0, R)} \overline{\varphi_{q}(x, k) f(x) d x,} \\
\left.\hat{f}_{q}^{(n)}=\left(f, \varphi_{q}^{(n)}\right)_{L_{2}(\Omega)} \quad \text { (function of } n=1, \cdots \cdots, N\right)
\end{array}
$$

are well-defined. While it is obvious that $\left\{\hat{f}_{q}^{(n)}\right\} \in l_{2}$ and the sum $\Sigma \hat{f}_{q}^{(n)} \varphi_{q}^{(n)}$ equals $E_{q}(0) f$, it is not at all clear from the outset whether the limit of (7.1) for $R \rightarrow \infty$ exists and has any meaning whatsoever.

Theorem 7.1. i) $\hat{f}_{q,}(k)$ converges in the mean to a unique $L_{2}\left(R^{3}\right)$-function

as $R$ tends to infinity and $\left\{\hat{f}_{q}^{(n)}\right\} \in l_{2}$.

$$
\hat{f}_{q}(k) \equiv\left(Z_{q} f\right)(k)
$$

ii) The operator $Z_{q}$ defined above is isometric from $\left(I-E_{q}(0)\right) L_{2}(\Omega)$ into $L_{2}\left(R^{3}\right)$ and the following Parseval formulas hold:

$$
\begin{gathered}
\|f\|^{2} L_{2}(\Omega)=\|\hat{f}\|^{2}{ }_{q L_{2}\left(R^{3}\right)}+\sum\left|\hat{f}_{q}{ }^{(n)}\right|^{2}, \\
(f, g)_{L_{2}(\Omega)}=\left(\hat{f}_{q}, g_{q}\right)_{L_{2}\left(R^{3}\right)}+\sum \hat{f}_{q}(n) g_{q}(n) .
\end{gathered}
$$

iii) Moreover, $Z_{q}$ maps onto $L_{2}\left(R^{3}\right)$, is invertible and for $f \in L_{2}\left(R^{3}\right)$ we have

$$
\left(Z_{q}{ }^{-1} f\right)(x)=(2 \pi)^{-3 / 2} \text { 1.i.m. } ._{R \rightarrow \infty} \int_{B(0, R)} \varphi_{q}(x, k) f(k) d k \in\left(I-E_{q}(0)\right) L_{2}(\Omega) ;
$$

the inversion formula for $f \in L_{2}(\Omega)$ is

$$
f(x)=\left(Z_{q}^{-1} \hat{f}_{q}\right)(x)+\Sigma \hat{f}_{q}^{(n)} \varphi_{q}^{(n)}(x),
$$

the last sum being convergent in the $l_{2}$-topology.

iv) Let $f \in L_{2}(\Omega)$. Then $f \in D\left(H_{q}\right)=D(H)$ if and only if $|k|^{2} \hat{f}_{q}(k) \in L_{2}\left(R^{3}\right)$ and 
$\left\{\mu_{q}{ }^{(n)} \hat{f}_{q}^{(n)}\right\} \in l_{2}$, in which case we have the following diagonal representation of $H_{q}$ :

$$
\left(H_{q} f\right)(x)=\left(Z_{q}^{-1} g\right)(x)+\Sigma \mu_{q}^{(n)} \hat{f}_{q}^{(n)} \varphi_{q}^{(n)}(x),
$$

where $g(k)=|k|^{2} \hat{f}_{q}(k)$.

In the course of the proof of the above theorem it will be shown that

$$
\left(\left(E_{q}(\mu)-E_{q}(0)\right) f, f\right)_{L_{2}(\Omega)}=\int_{|k|^{2} \leq \mu}\left|\hat{f}_{q}(k)\right|^{2} d k \quad(\mu \geq 0),
$$

which shows that no singular spectrum exists on the positive real line. It is also immediate from iii) and iv) above and Theorem 4.2 to see the similarity between $H_{q}$ in $\left(I-E_{q}(0)\right) L_{2}(\Omega), H$ and $H^{0}\left(=-\Delta\right.$ in all of $\left.R^{3}\right)$. Thus we have

Theorem 7.2. The (absolutely continuous) part in $\left(I-E_{q}(0)\right) L_{2}(\Omega)$ of $H_{q}$ is unitarily equivalent to $H$ and $H^{0}$ (in $L_{2}(\Omega)$ and $L_{2}\left(R^{3}\right)$ respectively). There exists no positive eigenvalue of $H_{q}$ and, moreover, the whole positive real line is occupied by the absolutely continuous spectrum of $H_{q}$.

We can carry out the proof of the above theorems by exactly the same method as we have used in $\S 9$ of [2] if we keep in mind only that we have to use $\varphi(x, k)$ instead of $e^{i k \cdot x}$ and replace the integration domain $R^{3}$ in $x$-space (not in $k$-space) by $\Omega$. Everything necessary has been prepared in $\S 5$.

But we should remark here that as regards the orthogonality of the eigenfunctions (Theorem 7.1, iii)), although it is possible to furnish a proof under our assumption (A) as in [2], it requires us to develop the theory of wave and scattering operators connecting $H$ and $H_{q}$, but if we assume in addition to (A) that $q(x)$ behaves like $O\left(|x|^{-3-h}\right)$ near infinity, then we can prove the orthogonality by the same technique as in [3] without any use of scattering theory, in which case the point is to be able to separate the eigenfunction $\varphi_{q}(x, k)$ in the form $e^{i k \cdot x}$ plus a term satisfying the radiation condition.

\section{References}

[1] M. Sh. Birman: On the spectra of singular boundary-value problems, Mat. Sbornik, 55 (97) (1961), 125-174. (In Russian).

[2] T. Ikebe: Eigenfunction expansions associated with the Schrödinger operators and their applications to scattering theory, Arch. Rational Mech. Anal., 5 (1960), 1-34.

[3] $T$. Ikebe: Orthogonality of the eigenfunctions for the exterior problem connected with $-\Delta$, Arch. Rational Mech. Anal., 19 (1965), 71-73.

[4] T. Kato: Fundamental properties of Hamiltonian operators of Schrödinger type, Trans. Amer. Math. Soc., 70 (1951), 195-211.

[5] O.D. Kellogg: Foundations of potential theory, Springer, 1929, Berlin.

[6] S. Mizohata: Sur l'analyticité de la fonction spectrale de l'opérateur $\Delta$ relatif au problème extérieur, Proc. Japan Acad., 39 (1963), 352-357.

[7] P.D. Lax and R.S. Phillips: Scattering theory, Bull. Amer. Math. Soc., 70 (1964), 130-142.

[8] P.D. Lax and R.S. Phillips: Scattering theory (book to be published).

[9] N.A. Shenk II: Eigenfunction expansions and scattering theory for the wave equation in an exterior domain, Arch. Rational Mech. Anal., 21 (1966), 120-150.

[10] Y. Shizuta: Eigenfunction expansion associated with the operator $-\Delta$ in the exterior domain, Proc. Japan Acad., 39 (1963), 656-660.

[11] P. Werner: Randwertprobleme der mathematischen Akustik, Arch. Rational Mech. Anal., 10 (1962), 29-66. 